\title{
A top surface liquid layer during membrane formation using vapor-induced phase separation (VIPS) —Evidence and mechanism of formation
}

\author{
P. Menut ${ }^{\text {a, } 1}$, Y.S. Su ${ }^{\text {c }}$, W. Chinpa ${ }^{\text {a }}$, C. Pochat-Bohatier ${ }^{a}$, A. Deratani ${ }^{b, *}$, \\ D.M. Wang ${ }^{c}$, P. Huguet ${ }^{b}$, C.Y. Kuo ${ }^{\text {d,e }}$, J.Y. Lai ${ }^{d, e}$, C. Dupuy ${ }^{a}$ \\ a UMR Cirad 016 Génie des Procédés d'Elaboration des Bioproduits, Université Montpellier 2, \\ CC 05, 2 Place E. Bataillon, 34095 Montpellier, France \\ ${ }^{\mathrm{b}}$ Institut Européen des Membranes, ENSC-UMII-CNRS, Université Montpellier 2, CC047, \\ 2 Place E. Bataillon, 34095 Montpellier, France \\ ${ }^{\mathrm{c}}$ Department of Chemical Engineering, National Taiwan University, Taipei, Taiwan \\ ${ }^{\mathrm{d}}$ Department of Chemical Engineering, Chung Yuan University, Chung-Li, Taiwan \\ ${ }^{\mathrm{e}} R \& D$ Center for Membrane Technology, Chung Yuan University, Chung-Li, Taiwan
}

Received 18 July 2007; received in revised form 31 October 2007; accepted 2 November 2007 Available online 19 November 2007

\begin{abstract}
The formation of a surface liquid layer on the top of membrane forming systems made of poly(ether-imide) (PEI) and $N$-methylpyrrolidone (NMP) was clearly demonstrated during water vapor-induced phase separation (VIPS) through several in situ investigation methods including optical microscopy and dynamic water contact angle measurements for a qualitative approach, and Raman confocal and FTIR microscopy for a quantitative one. A mechanism involving the shrinkage from the polymer-rich phase consecutively to the surface phase separation is proposed to account for the significantly high concentration of PEI in the surface liquid layer. The emergence of a surface liquid layer during the phase separation process is discussed in terms of implications on morphology of membrane fabricated using VIPS and how it contrasts with liquid-induced phase separation.
\end{abstract}

(C) 2007 Elsevier B.V. All rights reserved.

Keywords: Poly(ether-imide); Vapor-induced phase separation; Surface liquid layer; In situ analysis; Membrane morphology

\section{Introduction}

Manufacturing of polymeric membranes is mainly based on phase separation processing. Techniques such as temperatureand non-solvent-induced phase separation (TIPS and NIPS) have been developed to produce the demixing of a homogeneous polymer solution in two phases, a polymer-rich and a polymer lean phase. Phase separation originates in TIPS from heat transfer generated by a rapid cooling down of the polymeric solution and in NIPS from mass transfer produced by exchange occurring between solvent and non-solvent. These different phase separation methods have been reviewed by Mulder [1]. The final

\footnotetext{
* Corresponding author. Tel.: +334671491 20; fax: +33467149119.

E-mail address: andre.deratani@iemm.univ-montp2.fr (A. Deratani).

${ }^{1}$ Current address: ENSA Montpellier UMR IATE, 2 Place Viala, 34060 Montpellier, France.
}

membrane morphology is the result of the solidification of the polymer-rich phase that forms the membrane solid matrix displaying a more or less asymmetric porous structure considering the formulation and process parameters.

Actually there are different ways to induce demixing of a polymer solution by NIPS according the non-solvent physical state, i.e. liquid non-solvent-induced phase separation (LIPS) and vapor-induced phase separation (VIPS). The latter method, first patented in 1922 for the preparation of cellulose ester membrane [2], consists of the exposure of the cast film to a non-solvent containing atmosphere prior to immersion in a coagulation bath. During this process, the non-solvent (usually water) is introduced by the gas phase and its absorption by the polymer solution leads to the phase separation. Films with a cellular morphology are generally obtained using VIPS as shown for instance for polycarbonate/[dioxane/DMAc] [3], polysulfone/DMF [4], polysulfone/NMP [5], [chlorinated polyvinyl chloride/PVP]/[THF/n-butyl alcohol] [6] and poly(ether-imide) 
(PEI)/NMP [7,8] systems. It should be noted that VIPS can afford other kinds of morphologies as nodular and lace-like structures as obtained for the polyvinylidene fluoride/DMF system by Matsuyama et al. [9]. On the other hand, Wang et al. [10] demonstrated that it was possible by dissolving poly(4methyl-1-pentene) in mixed hydrophilic/hydrophobic solvents to confine the cellular structure in a single layer at the top of the film, whereas the remainder part of the membrane stayed dense.

In VIPS the water vapor exposure step is the key stage for the membrane structure formation. The water mass transfer penetration, which depends on the process parameters, is expected to control the morphology of the obtained films. This was demonstrated for the PEI/NMP system by adjusting the air relative humidity (RH) and temperature [7]. It was shown at $40^{\circ} \mathrm{C}$ that the film morphology changed from dense to cellular at $\mathrm{RH}$ of $27 \%$. Anisotropic membranes featuring a cell size gradient along the film cross-section were obtained for $\mathrm{RH}$ values higher than $27 \%$ whereas the cell and pore size decreased with RH. Although most commercially available membranes are fabricated using LIPS, VIPS can be regarded as a technique of interest to produce membranes of various kind of morphology including polymer films with ordered surface pattern [11], highly porous membranes [12], and surfaces with superhydrophobicity [13] and membranes without the macrovoid defaults commonly observed for membranes obtained by direct immersion in a coagulation bath [14]. Actually macrovoids have a positive effect on the permeation rate and a negative effect on the mechanical stability of the material entailing possible breakage during the permeation process. Depending on the application, the presence of macrovoids may have to be avoided. Although answers have been found, such as adjusting the composition of the dope solution and the coagulation bath [14-16], it remains one of the main problems in LIPS. It has been recently found that the formation of macrovoids can be successfully inhibited by incorporation of a stage of VIPS prior to immersion precipitation [17]. When solvent with low volatility and high affinity for water, such as NMP, is used to prepare polymeric hollow fibers, water in the air gap can be drawn to the polymer solution to induce phase separation (VIPS) before the membrane forming fiber is immersed in the coagulation bath to produce a rapid extraction of the solvent and the quenching of the structure. The formation of hollow fibers can therefore be considered as a process based on a coupling of VIPS and LIPS.

Another recent example is the fabrication of polymeric microsieves [18,19] called phase separation micromolding $(\mathrm{PS} \mu \mathrm{M})$. This very interesting method combines photolithography and phase separation to produce uniform and defect free microstructures. Polymer solutions were first cast into microfabricated silicon molds and then brought to phase separation. Phase separation occurred in two stages: VIPS where shrinkage and subsequent perforation of the film take place, helping also to prevent the macrovoid formation and LIPS where lateral shrinkage facilitates the release of the polymer replica from the mold [19].

Most of the studies available in literature give information on tailoring the structure of the membrane by using appropriate polymer/solvent formulations and by adjusting the process parameters (temperature, relative humidity, non-solvent exposure time) to reach optimal conditions and desired morphology. In addition, some authors have also proposed mathematical models to describe the mass transfer near the cast film/air interface $[9,20,21]$. These models can give predictions of profile concentrations but they are only valid for the homogeneous solution, until the onset of phase separation. The evolution of the mass transfer during the whole process is not completely understood, as well as the series of the mechanisms along the phase separation. Although, phenomenological explanations have been presented for the evolution of a forming-film consisting of PEI/NMP during VIPS [7], the best way to propose a clear description of the membrane formation appears to be "in situ" analysis performed at different scales during the phase separation process.

In this work, we focus on the forming-film surface evolution, which plays an important role in the structure formation of membranes using VIPS. During the stage of exposure to humid air, the air/polymer solution interface controls the mass transfer between the film and its environment, as well as the mass fluxes inside the forming-film itself. Also, demixing should be greatly affected by interfacial phenomena between the gas and liquid phases. Some of us have postulated in a previous paper [8] about the existence of a surface liquid layer mainly composed of volatile components after results obtained through a kinetic study of the overall mass transfer using gravimetric measurements. On the other hand, Wang et al. in an above-mentioned paper on a novel method for controlling the surface morphology of polymeric membranes [10] have suggested likewise that the polymer concentration near the air/polymer solution interface could be much lower than that in the bulk to explain the formation of a cellular surface structure.

The present report, using optical microscopy and contact angle measurements, shows evidence for the existence of such a surface liquid layer on top of forming membranes during VIPS stage. Information about its composition was obtained by adapting VIPS experiments to analytical instruments including Raman confocal and FTIR microscopy. A mechanism for the liquid layer formation is then proposed describing its initiation, growth and thinning. Finally, implication on the final membrane morphology is reported.

\section{Experimental}

\subsection{Materials}

The PEI used in this study was purchased from Aldrich (poly (bisphenol A-co-4-nitrophtalic anhydride-co-1,3-phenylene diamine), ref. $43,229-6)$. It was dried for $24 \mathrm{~h}$ at $170^{\circ} \mathrm{C}$ under vacuum, and stored under dry conditions before use. Anhydrous $N$-methyl-2-pyrrolidinone (NMP) from Aldrich (ref. 32,863-4) was used without any further purification and was taken out under nitrogen atmosphere for polymer solution preparation. The dope solutions were prepared at room temperature by stirring PEI pellets in NMP for 5 days. The obtained solutions were stored in a glove box where $\mathrm{RH}$ was kept below $7 \%$ and used within a week. 


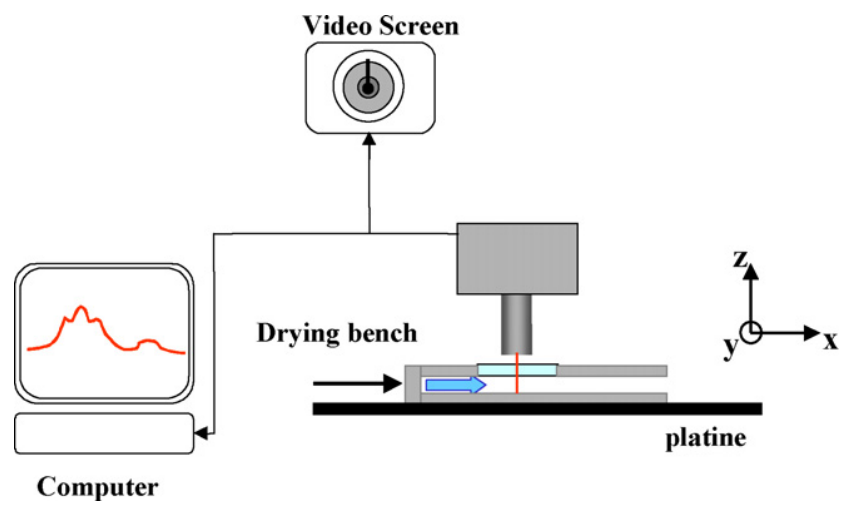

Fig. 1. Raman confocal spectrometry set-up for in situ analysis of the film surface composition.

\subsection{Membrane elaboration}

The dope solution was cast on a glass plate with a casting thickness of $250 \mu \mathrm{m}$ using an automatic coater (K Control coater, Erichsen). The casting operation was carried out at ambient temperature in a glove box in which RH was kept under $7 \%$. The cast film was then rapidly introduced into a drying bench at $40 \pm 0.5^{\circ} \mathrm{C}$ and brought into contact with a constant laminar air flow $\left(3.3 \mathrm{~L} \mathrm{~min}^{-1}\right.$ ) having $\mathrm{RH}$ monitored by bubbling air in a water column maintained at a specified temperature [7].

\subsection{Video-recording of the membrane surface}

The surface evolution of the membrane forming system in the drying bench was investigated using an optical video camera (mono CCD KAPPA 1/2 in.). Photographs of the film were taken at different times, and the shrinking of the surface area of the cast film was then quantified by using image processing software (image tool from UTHSCSA).

\subsection{Raman confocal spectrometry (RCS)}

A drying bench was specially designed to analyze composition evolution at the membrane surface with a RCS microscope during VIPS (Fig. 1). Air flow was optimized in order to operate in the same hydrodynamic conditions, so that the results obtained in the two benches could be compared. The RCS set-up was composed of a Labram 1B spectrometer (Dilor, Jobin-Yvon) with a $\mathrm{He} / \mathrm{Ne}(633 \mathrm{~nm})$ laser and equipped with an $x$-, $y$-motorized stage that allowed a manual control of the $z$-position for the stepwise scanning in the confocal measurements. The surface of the cast film (air/polymer solution interface side) was investigated by observation on a video screen. The laser beam, with a focalization cylinder of $1 \mu \mathrm{m}$ in diameter and $6 \mu \mathrm{m}$ in depth, was then focused on the film surface to scan Raman spectra. The mass ratio NMP to PEI at the focalized spot can then be determined at different times from the two characteristic peaks located at 3065 and $2925 \mathrm{~cm}^{-1}$, assigned to PEI and NMP, respectively. For each point, the ratio of the two peak areas (3060-3110 and 2800-3050 $\mathrm{cm}^{-1}$ ) was then used to correlate with the mass ratio of NMP to PEI using a calibration curve obtained for solutions having known PEI/NMP proportions. It should be noted that this technique does not allow the determination of the amount of water, as Raman spectroscopy is not sensitive enough to this molecule in the range of concentration studied.

The experiments were conducted at $25^{\circ} \mathrm{C}$. The variation of the film thickness during the VIPS stage was monitored by focalization the light beam both at the surface of the membrane forming system and at the polymer solution/glass plate interface.

\subsection{Contact angle measurement}

Water contact angle of the cast film was measured at $25^{\circ} \mathrm{C}$ $(\mathrm{RH}=55 \pm 5 \%)$ to characterize how the surface of the cast film changed during membrane forming. A water drop was introduced on the cast film, and the contour of the water drop was recorded by using an automatic interfacial tensiometer (Kyowa Interface Science, PD-VP). Analysis of the contour gave the water contact angle. It should be noted that the contour of the water drop on the cast film changed with time because of the miscibility of water with the solvent contained in the film. The contact angle reported in the present work is the initial contact angle, measured at $17 \mathrm{~ms}$ after the contact of the film with the water drop.

\subsection{FTIR microscopy}

A FTIR microscope (PerkinElmer, LE 186-0078) was used to analyze the mass ratio of NMP to water near the surface of the cast film at $25^{\circ} \mathrm{C}$ and humid air having $55 \pm 5 \% \mathrm{RH}$. A drop of PEI/NMP solution was placed in between two transparent $\mathrm{CaF}_{2}$ plates with a gap of $15 \mu \mathrm{m}$ formed by a Teflon spacer, through which humid air was introduced. IR beam was then focalized on a spot $(20 \mu \mathrm{m}$ in width and $300 \mu \mathrm{m}$ in length) in the cast film near the film-air interface, and the spectra obtained at different times were analyzed to characterize the ratio of NMP to water at that spot. Two characteristic peaks, at $1400 \mathrm{~cm}^{-1}(\mathrm{~N}-\mathrm{C}=\mathrm{O})$ and $3500 \mathrm{~cm}^{-1}(\mathrm{O}-\mathrm{H}$ stretching $)$, were identified as representing NMP and water, respectively. The ratio of the two peak heights was used to correlate with the mass ratio of NMP to water. Solutions of NMP/water with various compositions were prepared and their IR spectra were obtained. A calibration curve was then obtained by plotting the mass ratio of NMP to water versus the obtained ratio of the heights of the characteristic peaks of NMP and water. With this curve, the ratio of the height of the characteristic peaks in the IR spectra can be converted to the mass ratio of NMP to water.

\subsection{Membrane morphology}

Membranes were fractured in liquid nitrogen and coated with platinum before examination by using scanning electronic microscopy (Hitachi S-4500). The pore distribution of obtained membranes was achieved by using intrusive mercury porosimetry measurements (Micrometrics-Autopore II 9213). 


\section{Results and discussion}

\subsection{Existence of a surface liquid layer on cast film}

The results of gravimetric measurements reported in our previous studies [7,22] indicated that, when the cast film of PEI/NMP was in contact with humid air having a $\mathrm{RH}$ value higher than $27 \%$, the overall weight of the cast film increased with the film exposure time owing to the higher rate of water intake as compared to solvent evaporation. It is shown in the present work that, in association with the water intake from humid air, a liquid layer emerges on top of the cast film.

\subsubsection{Observation with an optical microscope}

The evolution of a PEI/NMP solution placed in between two plates during contact with humid air $\left(55 \pm 5 \% \mathrm{RH}\right.$ at $\left.25^{\circ} \mathrm{C}\right)$ was observed under an optical microscope. At first, the solution was transparent as shown in Fig. 2a. After some time, the amount of water intake was high enough (binodal being located for a water mass fraction of about $5 \%$ independently of the polymer concentration [22]) to bring about phase separation and the phase-separated region turned opaque at the air-solution interface, as shown in Fig. 2b. Following that, the phase-separated region was enlarged along with the occurrence of a surface liquid layer, which can be clearly seen in Fig. 2c. The thickness of the liquid layer firstly increased for a period of time and then decreased till it disappeared. This observation provides evidence for a liquid layer on the cast film which emerges due to the intake of water from humid air.
Although Fig. 2 gives clear indication of the existence of a surface liquid layer during membrane forming by VIPS, further evidence is still needed because the conditions for membrane forming under a microscope is not exactly the same as those normally used for preparation of membranes. The normal procedure is to cast the polymer solution on a glass plate; that is, the bottom of the cast film is in contact with the glass plate and the top is exposed to air. However, for clear observation under a microscope, the solution was placed in between two transparent plates and humid air was introduced through the small gap between them. The ratio of the surface area in contact with water vapor to the thickness of polymer solution was much less under the microscope than the normal membrane forming conditions. Further proof from in situ experiments should be acquired to clearly demonstrate that a surface liquid layer does emerge under the normal conditions of membrane forming.

\subsubsection{Measurement of the water contact angle of cast film}

A $16 \mathrm{wt} \%$ PEI/NMP solution was cast on a glass plate and exposed to the humid air $(55 \pm 5 \% \mathrm{RH})$ at $25^{\circ} \mathrm{C}$. The water contact angle of the film was measured after it was exposed to the humid air for various time periods. The change of film water contact angle with exposure time was thus determined and the results are presented in Fig. 3. It can be seen that the water contact angle decreased initially with exposure time, reached and stayed at zero for a certain period, and then increased with longer exposure time. It is not surprising to see the initial decrease in contact angle because the intake of water can obviously make the film more hydrophilic. However, the observation of zero contact (a)

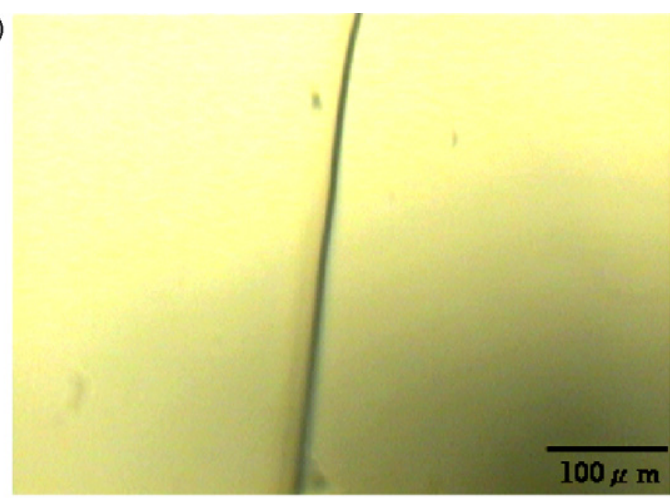

(b)

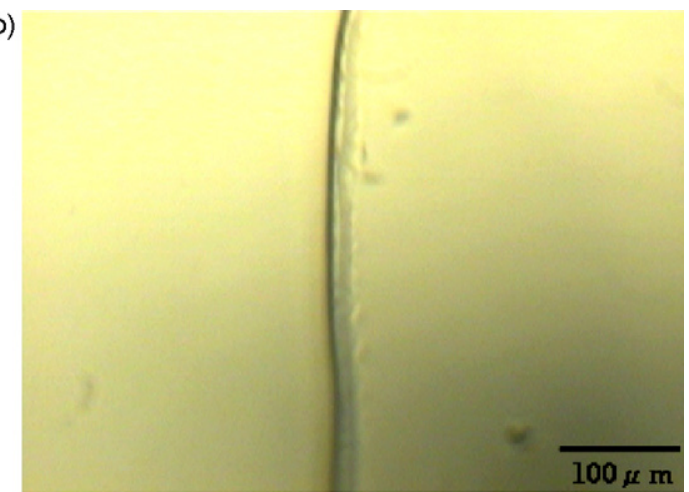

(c)

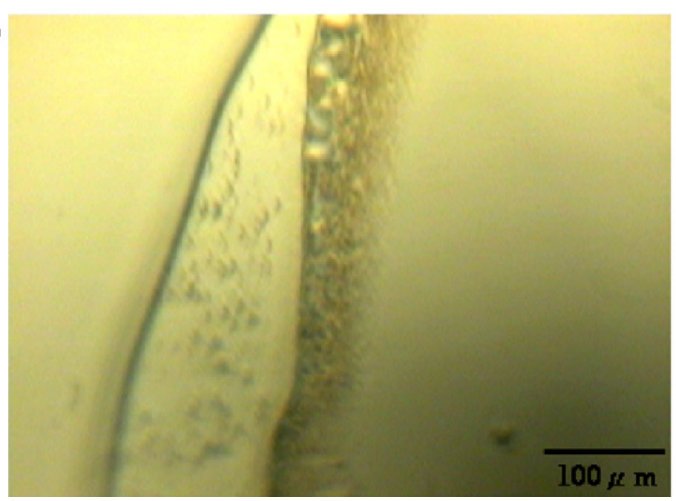

Fig. 2. Formation of a surface liquid layer observed under microscope (magnification $150 \times$ ): (a) before phase separation, (b) phase separation without forming liquid layer at the magnification scale of the microscope and (c) after the occurrence of liquid layer. 
angles needs more elaboration. Water was added in the $16 \mathrm{wt} \%$ $\mathrm{PEI} / \mathrm{NMP}$ solution and the resulted ternary solution was cast to form a film, of which the water contact angle was measured. It was observed that, although the addition of water made the water contact angle lower, it could not be lowered to zero. On the other hand, a liquid film without polymer (a film of NMP) had a zero water contact angle, believed to result from the high affinity of NMP for water. Actually it was found that the contact angle was zero for cast films containing less than $4 \mathrm{wt} \%$ of PEI. Therefore, it is believed that the observation of zero water contact angles indicated that the polymer concentration at the film surface was less than $4 \mathrm{wt} \%$, much lower than that in the bulk (16 wt\%). In other words, during membrane forming, there occurred a liquid layer near the film surface, and the polymer concentration in the layer could be lower than $4 \mathrm{wt} \%$. Obviously, the results of water contact angle confirm the emergence of a surface liquid layer on the cast film. It was also observed that the cast film was whitened, indicative of phase separation, after 4 min of exposure to the humid air. With this information and the data shown in Fig. 3, it is known that the surface liquid layer occurred after phase separation of the film, corresponding well to what was observed by optical microscope.

\subsection{Characterization of the surface liquid layer}

Figs. 2 and 3 give evidence confirming that a surface liquid layer formed when the cast film was exposed to humid air. Data presented in this section give the composition in the liquid layer that can provide information for understanding the formation of such a layer and clarifying its effect on membrane morphology.

\subsubsection{Raman confocal spectroscopy}

Changes in the film thickness and in the NMP/PEI ratio at the film surface were determined by using RCS during membrane forming in dried and humid air, with $\mathrm{RH}$ of 0 and $50 \%$ at $25^{\circ} \mathrm{C}$, respectively. These two conditions were selected to represent two clearly different macroscopic behaviors: under $0 \% \mathrm{RH}$, no water was available for the film to absorb and it was translucent all along the membrane forming process; whereas under $50 \%$ $\mathrm{RH}$, the film absorbed water and whitened after phase separation.

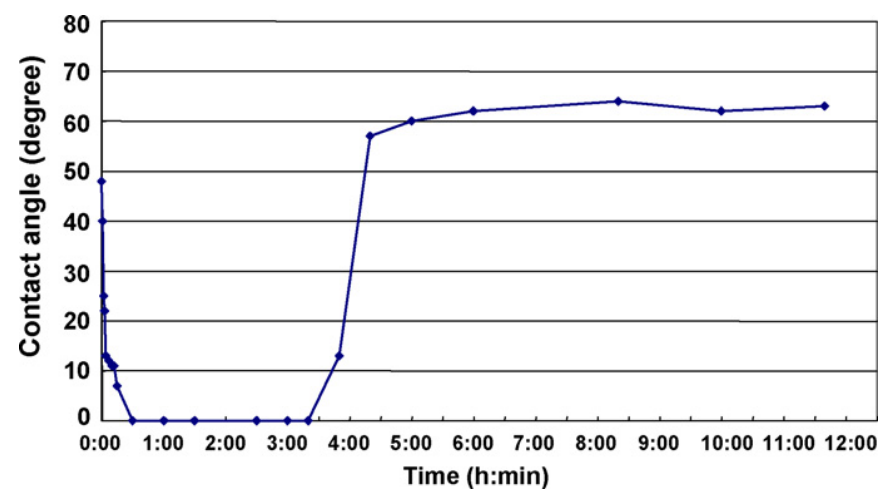

Fig. 3. Evolution of the water contact angle of a cast film vs. time during membrane forming by exposure of $16 \mathrm{wt} \% \mathrm{PEI} / \mathrm{NMP}$ solution to humid air with $50 \%$ $\mathrm{RH}$ at $25^{\circ} \mathrm{C}$.

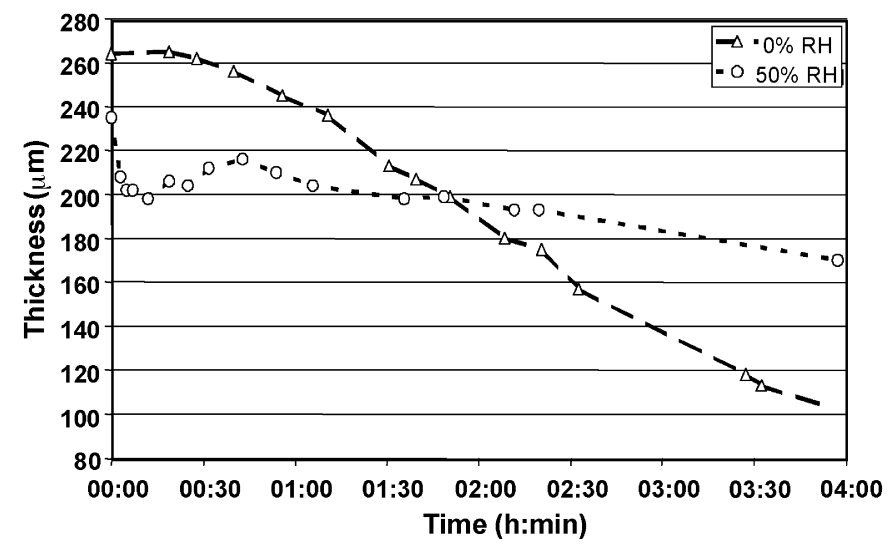

Fig. 4. Evolution of the film thickness vs. time during the first $4 \mathrm{~h}$ of VIPS under $0 \% \mathrm{RH}(\triangle)$ and $50 \% \mathrm{RH}(\bigcirc)$.

The prepared membranes were dense under dry air, and had a cellular structure below 50\% RH [22].

Reported in Fig. 4 are the results of film thickness at different exposure time. Under the dry condition, the film thickness decreased steadily with exposure time, in agreement with what was obtained in gravimetric measurement where the mass also steadily decreased owing to solvent evaporation [7,22]. On the other hand, under $50 \% \mathrm{RH}$, after an initial short period of decreasing thickness, an increase in the thickness was observed, and then followed by another period of decreasing. The trend observed is consistent with the evolution of film mass obtained from gravimetric measurement [22], except for the initial short period of decreasing thickness, which indicates the shrinkage of cast film and will be discussed later. The increase in film thickness corresponds to the increase in film mass, representing a period when the rate of water intake is higher than that of solvent evaporation. The long-term decrease in film thickness corresponds to the mass loss caused by the dominant rate of volatile component (NMP and water) evaporation over water intake. The results presented in Fig. 4 are also in agreement with what was observed by the optical microscope. It was observed under the microscope that the thickness of the surface liquid layer increased initially with the exposure time in humid air and decreased in the later period. Obviously, the occurrence and growth of the surface layer corresponds to the increase in film thickness.

After the position of the air-liquid interface was located, the NMP/PEI mass ratio at that point was determined by RCS. The results obtained under dry and humid conditions are presented in Fig. 5. The initial PEI concentration in the cast film was $16 \mathrm{wt} \%$, corresponding to a mass ratio of solvent to polymer of 5.25 , coherent with the results obtained by RCS in the first few minutes shown in Fig. 5. When the film was in contact with dry air, the solvent/polymer ratio at the interface decreased gradually, believed to be resulted from the evaporation of solvent during membrane forming. On the other hand, when the film was in contact with the humid air with $50 \% \mathrm{RH}$, the evolution of the solvent/polymer ratio had a totally different trend. The ratio increased up to a maximum of 18 after about $1 \mathrm{~h}$ of exposure, and then decreased with the exposure time. High sol- 


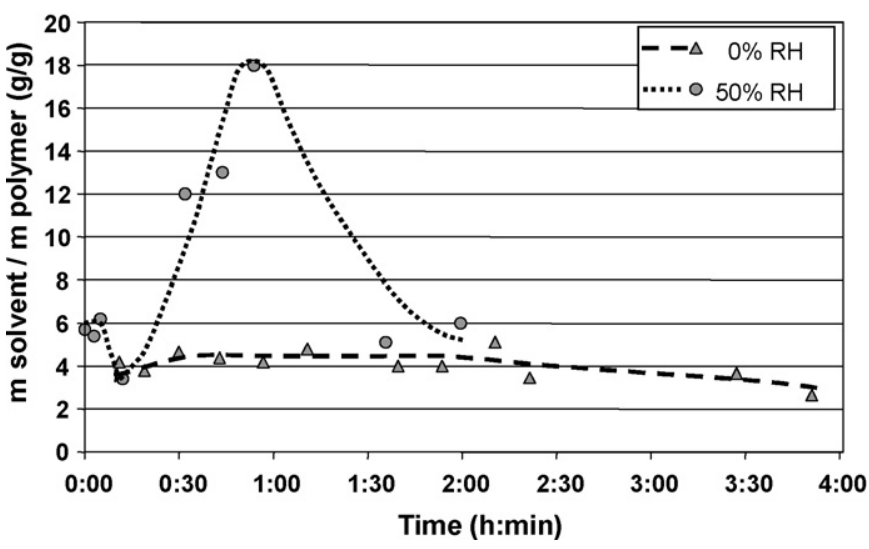

Fig. 5. Evolution of the solvent/polymer (NMP/PEI) ratio measured (Raman) at the surface of cast film vs. time during the first $4 \mathrm{~h}$ of VIPS under $0 \% \mathrm{RH}(\boldsymbol{\Lambda})$ and $50 \% \mathrm{RH}(\bullet)$.

vent/polymer ratio is indicative of low polymer concentration at the measured point. The results in Fig. 5 show that, when the cast film was exposed to humid air, the polymer concentration at the film surface first decreased and then increased. Fig. 5 not only provides evidence supporting the existence of a surface liquid layer, complying with what were shown in Figs. 2 and 3, but also gives quantitative information about the composition in the layer. The mass ratio of polymer to solvent in the surface layer reached a minimum of about 1/18 (5.6 wt $\%)$. It should be noted that in the above analysis water was yet included. After taking into account the presence of water in the surface layer, the polymer concentration would be lower than $5.6 \mathrm{wt} \%$. From the gravimetric measurement, it was observed that water was drawn from the humid air to the film. But it is not known how much water is present in the surface liquid layer. Raman spectroscopy cannot give any information about water. In the following data from FTIR microscopy are reported which can give the ratio of water to solvent in the surface layer.

\subsubsection{FTIR microscopy}

As shown in Fig. 2c, a liquid layer was observed under a microscope after phase separation of the polymer solution. IR beam was focalized at the air-liquid interface, and the spectrum obtained was analyzed to characterize the ratio of solvent (NMP) to water at the surface of the liquid layer. Presented in Fig. 6 is the dependence of the mass ratio of NMP to water on the exposure time. It can be seen that the ratio decreased with the exposure time, indicating higher water concentration in the liquid layer with longer exposure time. The results are reasonable since more water was drawn to the solution with longer exposure time. According to Fig. 6, the ratio of NMP to water in the liquid layer ranged from 7.2 to 5.1, showing that the liquid layer contained more NMP, about five to seven times as much as water.

Since the surface liquid layer resulted from the intake of water from the humid air, one might presume that water was present in a larger quantity at the surface, as compared to solvent. However, the results from FTIR microscopy show that water was only about $1 / 7$ to $1 / 5$ of NMP in the surface liquid layer. To make

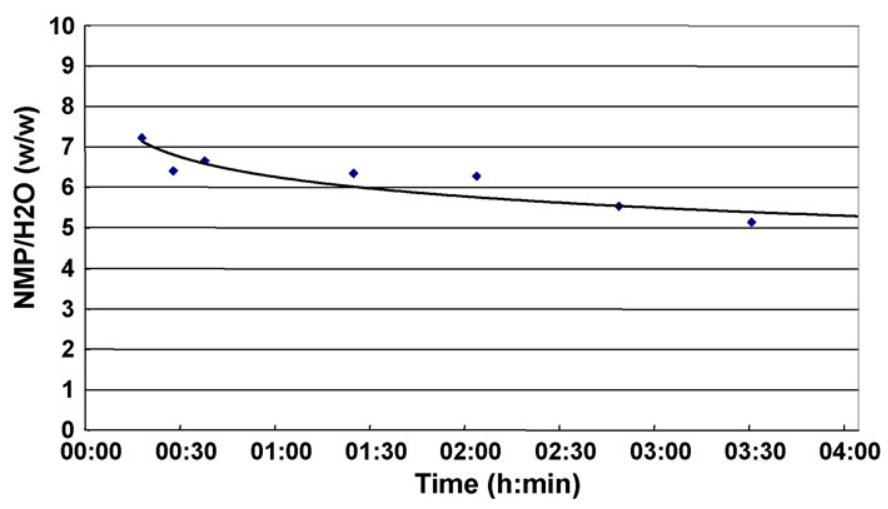

Fig. 6. Evolution vs. time of the solvent/water (NMP/water) ratio measured (FTIR) at the surface of cast film during membrane forming by VIPS under about $60 \% \mathrm{RH}$.

such a high NMP/water ratio, NMP must have migrated from the phase-separated region toward the surface liquid layer. The migration of NMP would have a dramatic effect on the final membrane structure, which will be discussed later in more detail.

With the results reported in Figs. 5 and 6, the composition at the surface of the liquid layer can be roughly estimated. For example, from Fig. 5, knowing the mass ratio of NMP to PEI could be as high as 18 , and taking the corresponding ratio of NMP to water as 6, estimated from Fig. 6, one can calculate the compositions at the surface of the liquid layer: $4.5 \mathrm{wt} \%$ of PEI, $13.5 \mathrm{wt} \%$ of water, and $82.0 \mathrm{wt} \%$ of NMP. Obviously the compositions change with time, but the above estimations give the reader a rough idea of the value of the composition of each component in the surface liquid layer. These data are in complete agreement with water contact angle measurements for which PEI concentrations lower than $4 \%$ give a zero value.

At $25^{\circ} \mathrm{C}$ a relative humidity of $50 \%$ corresponds to a water vapor pressure of $1584 \mathrm{~Pa}$. It is assumed that the thermodynamic equilibrium was reached after ca. $1 \mathrm{~h}$ when the ratio $\mathrm{m}$ solvent $/ \mathrm{m}$ polymer has the highest value in Fig. 5. From the composition of the surface liquid layer, the molar fraction of water can be estimated to be $X_{\mathrm{w}}=0.47$ neglecting the polymer contribution. It has to be noted that the corresponding water vapor pressure would be ca. $1350 \mathrm{~Pa}$ at the thermodynamic equilibrium for a binary NMP-water mixture. This result is in quite good agreement with the experimental data reported in Fig. 5. As can be seen water vapor condenses in the surface layer before a contact time of $1 \mathrm{~h}$ that is for a water vapor pressure below the applied relative water vapor pressure. Beyond this contact time, the evaporation of water and NMP by the air stream moves the equilibrium with time by decreasing the amount of the surface liquid layer.

\subsection{Mechanism of the formation of surface liquid layer}

\subsubsection{Initiation of the surface liquid layer}

It was clearly observed under a microscope that the surface liquid layer formed only after the cast film phase separated, as indicated in Fig. 2. Since the gelation of polymer solution after phase separation would retard water diffusion into it, a reasonable conjecture on the initiation of the surface layer is the 


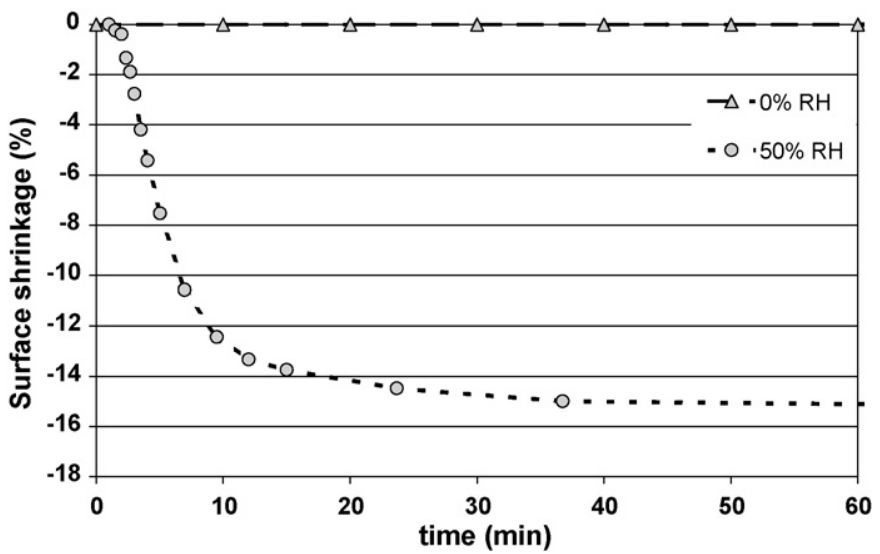

Fig. 7. Degree of the film shrinkage during membrane forming by VIPS under $0 \% \mathrm{RH}(\boldsymbol{\Delta})$ and $50 \% \mathrm{RH}(\bullet)$

accumulation of water on the film surface as a consequence of the higher rate of water intake from the air than diffusion into the film. The accumulated water on the surface, due to its high affinity for NMP, could then extract the NMP out of the film, which can well account for the high NMP/water ratio in the layer, as shown in Fig. 6. However, this proposed mechanism cannot satisfactorily explain the results shown in Fig. 5. Since water is a strong non-solvent for PEI, the accumulated water could not extract the polymer into the surface layer; even if some PEI migrated with NMP into the layer, the amount was supposed to be small. Nevertheless, according to Fig. 5, the weight ratio of PEI to NMP in the layer was at least $5 \mathrm{wt} \%$ and could be about $9 \mathrm{wt} \%$ or even higher. Therefore, accumulation of water on the film surface seems not to be a suitable mechanism. It will be shown below that film shrinkage caused by polymer contraction is more appropriate to account for the initiation of the surface liquid layer.

With a video camera the image of the cast film was recorded and the film area was estimated. The area difference was calculated between the initial film and the one at a given time during membrane forming, and its ratio to the initial area was defined as the degree of film shrinkage at that time. Fig. 7 shows the degree of film shrinkage in the first hour of membrane forming. For the case of 50\% RH, no obvious shrinkage was observed in the initial period of membrane forming. Shrinkage was observed only when the film was exposed to humid air for longer than $4 \mathrm{~min}$, as the cast film whitened. On the other hand, when the air was dry, the cast film remained transparent during membrane forming and no film shrinkage was observed. The results indicate that the film shrinkage was strongly related to whether or not phase separation occurred. Little film shrinkage was observed without phase separation, and a degree of film shrinkage of about $15 \%$ was observed $20 \mathrm{~min}$ after phase separation.

The film shrinkage was associated with movement of polymer away from the film periphery and toward the center. A possible mechanism for the polymer movement is that the absorbed water at the surface makes the polymer activity higher due to the decrease of the quality of solvent (water is a non-solvent for PEI) and thus generates a driving force for polymer to diffuse away from the surface and into the bulk. Another possible mech-

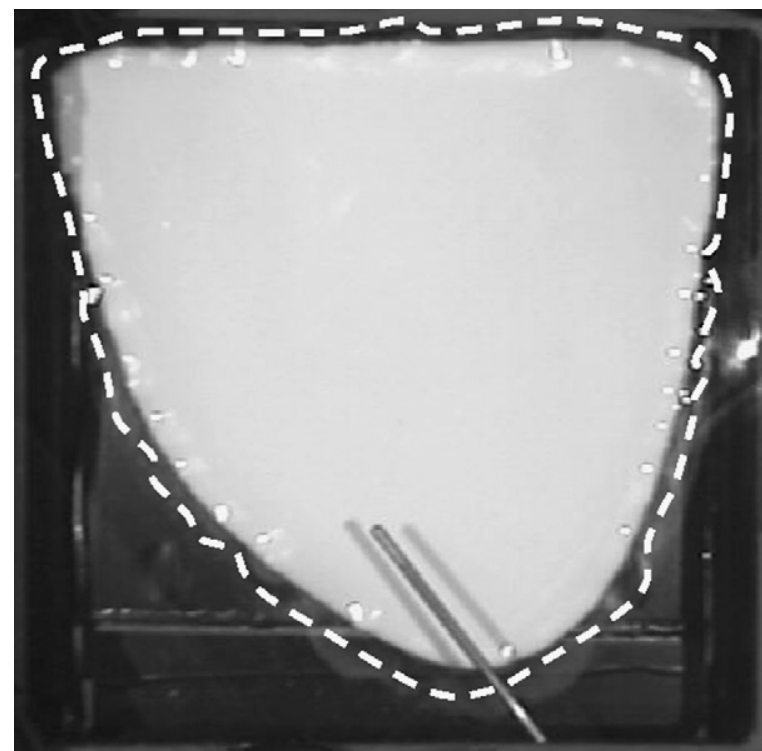

Fig. 8. Photograph of a cast film under $50 \%$ RH, after about $30 \mathrm{~min}$. The white dotted lines represent the initial film periphery.

anism is the contraction of the polymer-rich phase after phase separation, which tends to pull the polymer together toward the bulk, like gel syneresis. The distance of polymer diffusion was estimated to deduce if diffusion is a reasonable mechanism. The diffusion distance can roughly be calculated by $\sqrt{D t}$, where $D$ denotes the diffusion coefficient and $t$ represents the time allowed for diffusion. A typical diffusion coefficient in concentrated solution is lower than $10^{-6} \mathrm{~cm}^{2} / \mathrm{s}$, and the diffusion distance for a diffusion time of $30 \mathrm{~min}$ would thus be less than $0.04 \mathrm{~cm}$. Besides, the distance of the movement of film periphery due to shrinkage is estimated by locating the film peripheries in the recorded video images. As shown in Fig. 8, the film periphery after phase separation can easily be located (the boundary of the white region) and the dotted line represents the initial film periphery. It can then be estimated by using Fig. 8 that after 30 min of exposure to humid air the distance of shrinkage was about $0.5 \mathrm{~cm}$, much longer than the estimated diffusion length. The above analysis suggests that polymer diffusion might not be the major mechanism responsible for the shrinkage of polymer. Therefore, it seems that the contraction of the polymer-rich phase would be a more reasonable mechanism to account for the film shrinkage.

It should be noted that the shrinkage of cast films was accompanied by the emergence of a liquid layer on the film periphery. A translucent liquid layer actually is located between the whitened film and the dotted line in Fig. 8. Such a liquid layer was not observed when RH was lower than $27 \%$, below which the film did not turn white and shrink at all. It is known that, in gel syneresis, contraction of gel leads to exudation of liquid at surface. A similar mechanism can link the emergence of a liquid layer with the contraction of polymer-rich phase and shrinkage of cast film.

The analysis described in the preceding paragraph was based on the observation of the horizontal shrinkage of cast film. Similar shrinkage also occurred in the vertical direction, the direction across the casting film, which can be verified by the data of film 
(a)

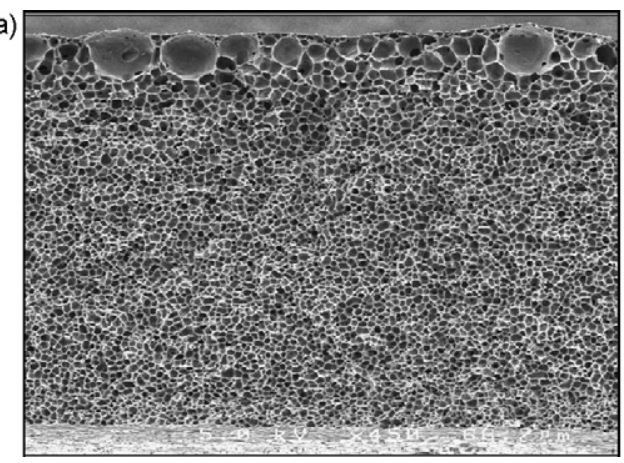

(b)

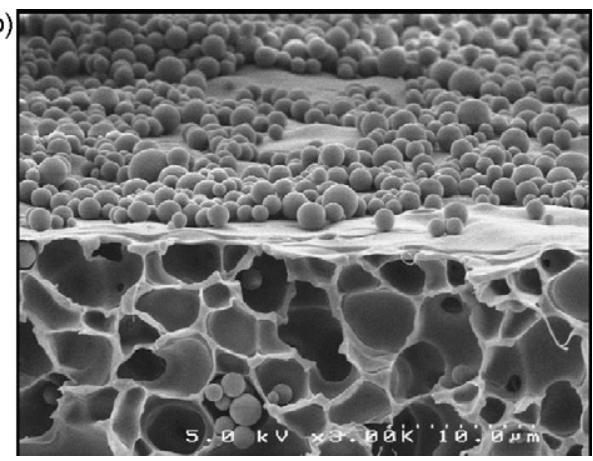

Fig. 9. SEM photograph of (a) total and (b) top cross-section of a membrane prepared by VIPS with a 16 wt $\%$ PEI/NMP solution under $50 \%$ RH.

thickness. Fig. 4 demonstrates that the film thickness decreased about $30 \mu \mathrm{m}$ after the film was in contact with humid air for about $10 \mathrm{~min}$. The original film thickness was $250 \mu \mathrm{m}$ and a decrease of $30 \mu \mathrm{m}$ in thickness indicates a degree of shrinkage of about $12 \%$. The results presented in Fig. 7 show that the horizontal shrinkage at 10 min was about $13 \%$, similar to the degree of shrinkage in film thickness. The above results indicate that the degrees of shrinkage are very close in the horizontal and vertical directions. Therefore, similar to the emergence of the liquid layer shown in Fig. 8 on the periphery of the cast film, a liquid layer also emerged on the surface of the film via the mechanism of contraction of polymer-rich phase, like gel syneresis.

\subsubsection{Growth of the surface liquid layer}

In the preceding section, it is proposed that the contraction of polymer after phase separation expels liquid at the surface. We can reasonably consider that it is only small quantities of polymer solution which are expelled from the bulk. The NMP contained in the exuded liquid can then draw water from humid air, resulting in increasing thickness of liquid layer (Fig. 4) and decreasing NMP/water ratio (Fig. 6), with longer exposure time to humid air. In the meanwhile, the intake water can extract more NMP by percolation from the phase-separated region to the liquid layer, which also contributes to the growth of the liquid layer and can account for the increasing NMP/PEI ratio (Fig. 5). The polymer is considered as motionless compared to the mass transfer of NMP and water so that the polymer concentration is lowered by the water and NMP intakes in the surface liquid layer. The water intake rate decreases with exposure time until the chemical potential of water in the liquid phase is equal to that in the gas phase. At that time the thickness of the liquid layer reaches a maximum value. Longer exposure time will result in decreasing of the liquid layer thickness (Fig. 4) and of the ratio of NMP/PEI (Fig. 6), corresponding to a drying stage and evaporation loss of NMP. It has to be noted that, during the drying stage, the rate of NMP extracted from the phase-separated region to the liquid layer should be lower than the NMP evaporation rate, so that drying of the liquid layer becomes possible.

\subsection{Implications of the existence of the surface liquid layer}

Since the surface liquid layer controls the mass transfer at the air film-forming interface, it has interesting effects on membrane morphology. In the following section, it is shown that the liquid layer is strongly related to the gradient in cell and pore size across the membrane and the occurrence of nodules and pinholes on top of membrane surface.

\subsubsection{Cell and pore size gradient across a membrane}

It has been reported $[7,22]$ that PEI membranes, prepared by VIPS from a $16 \mathrm{wt} \% \mathrm{PEI} / \mathrm{NMP}$ dope solution, have larger cells near the membrane surface and smaller ones near the bottom, as can be seen in Fig. 9a. The cellular structure is believed to arise by phase separation taking place through a nucleation and growth process [7].

Interconnecting pores between cells were measured by mercury intrusion porosimetry (Fig. 10). The obtained data present a bimodal distribution with two pore size populations, having an average diameter of about 0.48 and $1 \mu \mathrm{m}$, respectively. The volume reached by mercury is much smaller for the larger pores (ca. 10\%) than that offered by the smaller ones reflecting the distribution of cells shown by the SEM micrograph of membrane cross-section (Fig. 9a).

All these results are focused on the presence of anisotropy in the membrane morphology, and it is relevant to associate the pore size distribution with the evolution of the cell size. The larger cells at the top membrane surface possess bigger pore size than the small cells which represents the main part of the average cell size of the whole membrane. Such observations can be reasoned by the distribution of polymer and solvent in association with the emergence of the liquid layer.

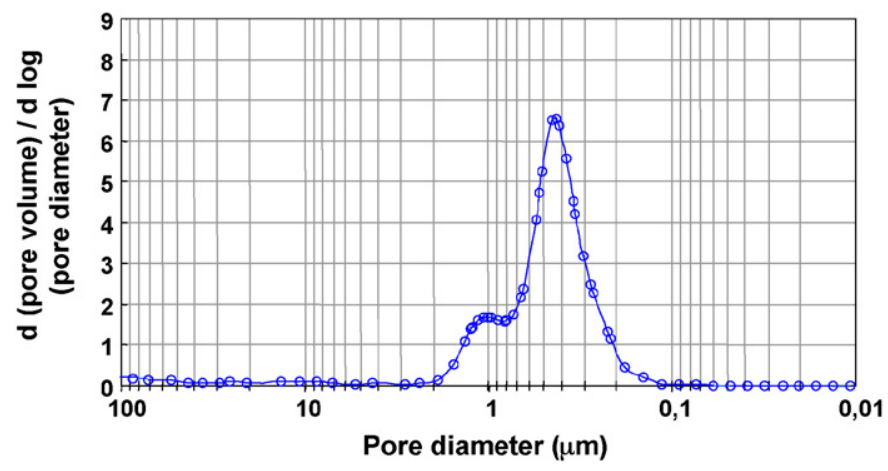

Fig. 10. Pore size distribution as obtained by mercury intrusion into a membrane prepared by VIPS with a $16 \mathrm{wt} \% \mathrm{PEI} / \mathrm{NMP}$ solution under $50 \% \mathrm{RH}$. 
The phase inversion process is usually divided into two categories: dry and wet processes. For the VIPS process, since the cast film is not immersed in the coagulation bath, it belongs to the category of dry process. However, owing to the emergence of a surface liquid layer, the cast film is actually in contact with liquid, similar to the wet process (LIPS). The surface liquid layer extracts the solvent out of the cast film, just like the wet process. The solvent concentration in the coagulation bath, for a typical wet inversion process, is very low because of the high volume ratio of the coagulation bath to the cast film. However, for the VIPS process, since the liquid layer is thin compared to the cast film and the evaporation rate of the NMP out of the liquid layer is low, the solvent migrates toward the membrane surface top layer and makes its concentration higher than that in the bulk, as discussed above. Therefore, the existence of the surface liquid layer generates a solvent gradient across the cast film, resulting in higher concentration near the surface and lower one near the bottom, along with lower polymer concentration near the surface and higher one near the bottom. The polymer concentration directly influences the viscosity of the solution, as a result the coarsening of droplets is generated more easily in the top layers of the membrane where the viscosity is lower than in the deeper ones. Cell growth, due to coalescence, is achieved with more delay in the top layers since the continuous polymer-rich phase reaches the glass transition concentration later than in the bulk of the membrane as already discussed in previous papers [7,22].

\subsubsection{Nodules on top of membranes}

Although the polymer concentration could be low in the surface liquid layer, it was not zero as shown in Fig. 5. With polymer in the surface liquid layer, phase separation would still occur when enough water was drawn into the layer. From the ternary diagram described in Ref. [22], it is known that a water concentration of about 5\% induces the binodal demixing. Because the polymer concentration in the liquid layer was low, the structure in this region resulted from phase separation might be different from that in the bulk of the cast film. Careful examination of the PEI membrane prepared via VIPS with a complete drying, as indicated in Fig. 9b, shows that polymer beads existed on top of the spongy structure. These nodules are usually not observed on the surface membrane if the cast film is immersed after the period of humid air exposure, but they are dispersed in the coagulation bath. It is believed that the emergence of the surface liquid layer can give a reasonable explanation for the occurrence of the nodules on top of the membrane surface. Fig. 2c shows tiny droplets in the surface liquid layer indicating that a demixing process occurred. Owing to the low polymer concentration in the surface liquid layer, secondary phase separation could occur via nucleation and growth of the polymer-rich phase, resulting in polymer beads. The proposed mechanism still needs further investigation to be confirmed; however, it seems quite probable that these nodules are remains of the surface liquid layer.

\subsubsection{Surface skin and pinholes}

It can be seen in Fig. $9 \mathrm{~b}$ that a dense surface skin was formed on the top of the large cell layer. The presence of this unexpected skin can be accounted for by the nucleation and growth mech-

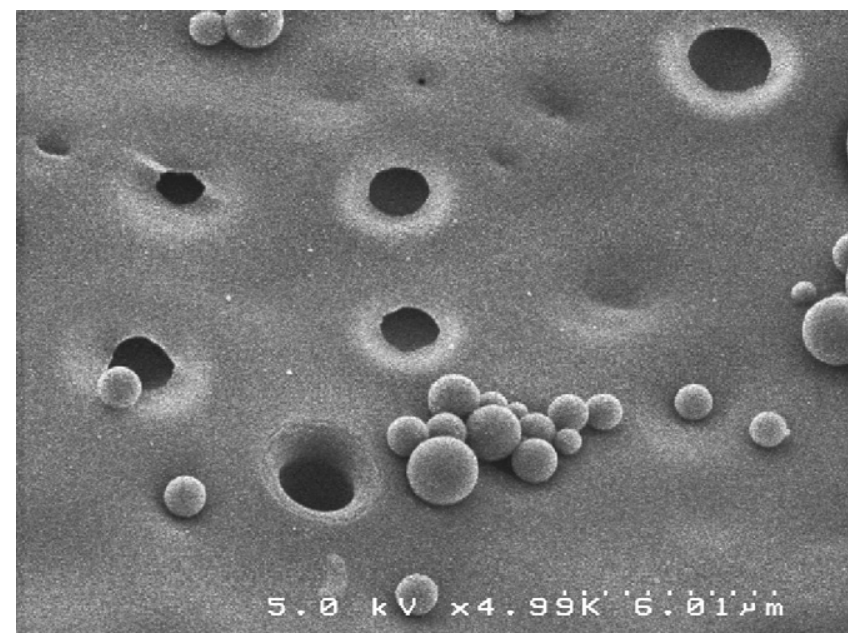

Fig. 11. SEM photograph of the top surface of a membrane prepared by VIPS with a $16 \mathrm{wt} \%$ PEI/NMP solution under 50\% RH.

anism of phase separation as follows. Cell size develops more easily by coalescence near the surface owing to a local lower viscosity as explained in Section 3.4.1. Moreover, it was demonstrated that the liquid layer mainly consists of NMP. Regarding its strong interaction with PEI, the gelation of the polymer-rich phase on the top surface could be considered to occur at longer times than for the other membrane layers. It is then believed that the gelation delay enables the formation of closed cells resulting in the formation of a dense top skin.

A deeper examination of the membrane surface in Fig. 11 shows polymer nodules and large round shaped pores, as well. Formation of such pinholes could arise from incomplete closure of cells during coalescence. This mechanism can be observed in the membrane bulk yielding interconnecting pores between the cells (Fig. 9b). Another plausible explanation concerns the shrinking phenomenon. Actually according to our proposed mechanism, the polymer solution is supposed to be expelled and forced to pass through the surface skin layer since film shrinkage takes place after the phase separation starts (Fig. 7). Then we assume that this phenomenon should create craters giving rise to pinholes in the final membrane structure. However, it must be kept in mind that these pores are formed just after surface phase separation. For this reason they might only exist at earlier stages because of the surface liquid mainly consisting of NMP.

Pinholes likely might come from incomplete coalescence and expelling effects and it seems difficult without further studies to decide which one could be predominant. Regardless of the mechanism, the presence of a dense skin and pinholes at the surface is due to the emergence of the surface liquid layer. As it is believed that these structures should play a significant role in the performances of the resulting membrane, we will develop in a further report ways in which to control their formation.

As a concluding remark, expelling of polymer solution related to mechanical constraints during film shrinkage might be a general mechanism occurring during membrane preparation using any phase separation technique especially when an evaporation stage is performed. This assumption is supported by several studies found in literature that were reported on the 
formation of polymer nodules during LIPS (see for instance [23-25]). Their occurrence and that of large surface pores, also, are directly related to the dope solution viscosity controlled by either the polymer molecular weight [24] or the polymer concentration $[24,25]$. Similar results were observed with the studied system (data not shown) [26]. The more detailed report deals with the preparation of cellulose acetate membrane using methyl acetate as solvent and 2-propanol as precipitant [24]. The authors demonstrated that the so-called filter dust produced during the membrane fabrication consisted of chains with a lower molecular weight than those in the dope solution. They postulated that expelling of the polymer lean phase enriched by fractionation with short chain component which takes place during phase separation is a reasonable explanation for the observed production of polymer nodules. Size exclusion chromatography of PEI particles formed in our system did not show such a marked decrease of the polymer molecular weight compared to that of the starting polymer. However, it was noted that the polymolecularity index is slightly reduced indicating that a fractionation occurred on to a small extent. The observed loss of low molecular weight compounds likely due to the high NMP concentration in the surface liquid layer, might balance the loss of high molecular weight compounds during phase separation yielding a mean average molecular weight for the particles similar to that of the starting polymer solution.

\section{Conclusion}

In this work, we investigated several methods to disclose the formation of a surface liquid layer during membrane elaboration using VIPS. Results obtained with optical microscopy and water contact angle measurements clearly demonstrated the existence of a thin layer on top of the forming membrane. According to the results obtained from RCS and FTIR microscopy analysis for the top surface composition of the cast film, the liquid layer appears after the phase separation and is likely to be initiated by the expulsion of a small amount of polymer solution consecutive to the shrinkage of the polymer-rich phase by a mechanism comparable to gel syneresis.

From our experience, a liquid layer seems to exist in all the VIPS systems. Once the interfacial water adsorption rate from the air becomes higher than the water mass transfer rate into the cast film, the liquid layer appears. Shrinkage of the cast film would be a sufficient condition to yield a liquid layer, because the water mass transfer rate into the cast film would be inhibited by the occurrence of a higher mass-transfer-resistance surface layer (skin layer) after the shrinkage.

By its location at the air-facing interface, the liquid layer acts as an intermediate media for the mass transfer in solvent and nonsolvent exchanges and strong implications are expected on the final membrane morphology prepared using VIPS. For instance in the studied system, it is the origin of the bigger cells observed near the air-facing interface and also entailing the presence of polymer nodules and pinholes on the top surface.

Actually the liquid layer could explain the difference observed between the morphology obtained with LIPS and VIPS, especially related to the macrovoid formation, which is strongly restricted during VIPS process. Our data demonstrate that the surface liquid layer emergence can be associated with the phase separation mechanism. The main difference comes from the composition of the liquid layer that is in contact with the membrane forming system basically consisting of non-solvent for LIPS and solvent for VIPS.

\section{Acknowledgements}

We are grateful to NSC Taiwan and CNRS France for their financial support through an exchange program of scientists.

\section{References}

[1] M.H.V. Mulder, Preparation of synthetic membranes, in: Basic Principles of Membrane Technology, 2nd ed., Kluwer Academic Publishers, Dordrecht, 1996, pp. 54-95 (Chapter III).

[2] R. Zsigmondy, W. Bachmann, Filter and method of producing same, US Patent 1,421,341 (1922).

[3] M. Bodzek, J. Bohdziewics, Porous polycarbonate phase-inversion membranes, J. Membr. Sci. 60 (1991) 25.

[4] M.-J. Han, D. Bhattacharyya, Changes in morphology and transport characteristics of polysulfone membranes prepared by different demixing conditions, J. Membr. Sci. 98 (1995) 191.

[5] H.C. Park, Y.P. Kim, H.Y. Kim, Y.S. Kang, Membrane formation by water vapor induced phase separation, J. Membr. Sci. 156 (1999) 169.

[6] J.S. Kang, K.Y. Kim, Y.M. Lee, Preparation of microporous chlorinated poly(vinylchloride) membrane in fabric and the characterization of their pore sizes and pore-size distributions, J. Appl. Polym. Sci. 86 (2002) 1195.

[7] H. Caquineau, P. Menut, A. Deratani, C. Dupuy, Influence of the relative humidity on film formation by vapour induced phase separation, Polym. Eng. Sci. 43 (2003) 798.

[8] P. Menut, C. Pochat-Bohatier, A. Deratani, C. Dupuy, S. Guilbert, Structure formation of poly(ether-imide) films using non-solvent vapour induced phase separation: relationship between mass transfer and relatice humidity, Desalination 145 (2002) 11.

[9] H. Matsuyama, M. Teramoto, R. Nakatani, T. Maki, Membrane formation via phase separation induced by penetration of non-solvent from vapor phase. I. Phase diagram and mass transfer process, J. Appl. Polym. Sci. 74 (1999) 159.

[10] D.M. Wang, T.T. Wu, F.C. Lin, J.Y. Hou, J.Y. Lai, A novel method for controlling the surface morphology of polymeric membranes, J. Membr. Sci. 169 (2000) 39.

[11] M. Srinivasarao, D. Collings, A. Philips, S. Patel, Three-dimensionally ordered array of air bubbles in a polymer film, Science 292 (2001) 79.

[12] I.F. Wang, J.F. Ditter, R. Zepf, Highly porous polyvinylidene difluoride membranes, US Patent 6,146,747 (2000).

[13] N. Zhao, J. Xu, Q. Xie, L. Weng, X. Guo, X. Zhang, L. Shi, Fabrication of biomimetic superhydrophobic coatings with a micro-nano-binary structure, Macromol. Rapid Commun. 26 (2005) 1075.

[14] W. Albrecht, Th. Weigel, M. Schossig-Tiedemann, K. Kneifel, K.-V. Peinemann, D. Paul, Formation of hollow fiber membranes from poly(ether imide) at wet phase inversion using binary mixtures of solvents for the preparation of the dope, J. Membr. Sci. 192 (2001) 217.

[15] H. Strathmann, K. Kock, P. Amar, R.W. Baker, The formation mechanism of asymmetric membranes, Desalination 16 (1975) 179.

[16] D.T. Clausi, W.J. Koros, Formation of defect-free polyimide hollow fiber membranes for gas separation, J. Membr. Sci. 167 (2000) 79.

[17] H.A. Tsai, C.Y. Kuo, J.H. Lin, D.M. Wang, A. Deratani, C. Pochat-Bohatier, K.R. Lee, J.Y. Lai, Morphology control of polysulfone hollow fiber membranes via water vapor induced phase separation, J. Membr. Sci. 278 (2006) 390

[18] L. Vogelaar, J. Barseman, C.J.M. Van Rijn, W. Nijdam, M. Wessling, Phase separation micromolding, Adv. Mater. 15 (2003) 1385. 
[19] M. Gironès, I.J. Akbarsyah, W. Nijdam, C.J.M. van Rijn, H.V. Jansen, R.G.H. Lammertink, M. Wessling, Polymeric microsieves produced by phase separation micromolding, J. Membr. Sci. 283 (2006) 411.

[20] V.P. Khare, A.R. Greenberg, W.B. Krantz, Vapor-induced phase separation-effect of the humid air exposure step on membrane morphology. Part I. Insights from mathematical modeling, J. Membr. Sci. 258 (2005) 140.

[21] Y. Yip, A.J. McHugh, Modeling and simulation of nonsolvent vapourinduced phase separation, J. Membr. Sci. 271 (2006) 163.

[22] A. Ripoche, P. Menut, C. Dupuy, H. Caquineau, A. Deratani, Poly (etherimide) membrane formation by water vapour induced phase inversion, Macromol. Symp. 188 (2002) 37.
[23] S.-C. Fan, Y.-C. Wang, C.-L. Li, K.-R. Lee, D.-J. Liaw, H.-P. Huang, J.-Y. Lai, Effect of coagulation media on membrane formation and vapor permeation performance of novel aromatic polyamide membrane, J. Membr. Sci. 204 (2002) 67

[24] J. Eckelt, S. Loske, M.C. Gonçalves, B.A. Wolf, Formation of microand nano-spheric particles (filter dust) during thee preparation of cellulose acetate membranes, J. Membr. Sci. 212 (2003) 69.

[25] V. Kaiser, C. Stropnik, V. Musil, M. Brumen, Morphology of solidified polysulfone structures obtained by wet phase separation, Eur. Polym. J. 43 (2007) 2515.

[26] W. Chinpa, PhD Thesis, University Montpellier 2, France, March 2006. 